\section{Los riesgos del uso inadecuado de Internet y las redes sociales. Conocimiento y percepción de riesgos en jóvenes a través de la historia de vida}

https://doi.org/10.17345/ute.2019.1

\author{
Elsa Calvarro Castañeda, Diego Galán Casado, Olvido Andujar Molina \\ Rebut: 04/07/2019 Acceptat: 15/07/2019
}

\begin{abstract}
Resumen
La presente investigación busca determinar el grado de conocimiento que tienen los jóvenes sobre la navegación en Internet y la utilización de las redes sociales y sus riesgos, así como la necesidad de educar en esta materia a nivel formal. Este trabajo se inserta dentro de la preocupación social emergente acerca de los riesgos potenciales que sufren los jóvenes, derivados de la utilización de las TIC. De este modo, utilizando la metodología biográfico-narrativa, se han obtenido los relatos vitales de diez estudiantes entre 18 y 25 años para descubrir su conocimiento sobre las TIC y sus riesgos, su percepción sobre la formación que han recibido en esta materia y la necesidad de que se eduque de manera formal desde las aulas. Los resultados obtenidos muestran un uso elevado en la conexión a Internet y las redes sociales, la utilización del smartphone como dispositivo fundamental de conexión, la utilización de herramientas para mantener contacto con otras personas, subir y visualizar fotografías, la ausencia de conocimiento específico sobre las implicaciones legales de un mal uso de Internet y redes sociales, así como la necesidad de educar en las aulas a lo largo de toda la etapa educativa.
\end{abstract}

Palabras Clave: riesgos, Internet, redes sociales, jóvenes, historias de vida

\begin{abstract}
This research seeks to determine the degree of knowledge that young people have about Internet browsing and the use of social networks and their risks, as well as the need to educate on this matter at a formal level. This work is inserted within the emerging social concern about the potential risks that young people can suffer, derived from the use of ICT. In this way, using the biographical-narrative methodology, the vital accounts of ten students between 18 and 25 years old have been obtained to discover their knowledge about ICT and its risks, their perception of the training they have received in this area and the need to be formally educated at school. The results obtained show a high use in the connection to Internet and social networks, the use of the smartphone as a fundamental connection device, the use of tools to maintain contact with other people, upload and view photographs, the absence of specific knowledge about the legal implications of misuse of the Internet and social networks, as well as the need to educate in the classrooms throughout the entire educational stage.
\end{abstract}

Keywords: risks, Internet, social networks, young people, life stories 


\section{Introducción}

La aparición a mediados de los años 90 de las TIC produjo una verdadera revolución social, debido a los cambios y avances que generaron en nuestra sociedad (Berríos y Buxarrais, 2005). Han supuesto un desarrollo tecnológico imparable que está generando un profundo e intenso impacto en todos los ámbitos y niveles de la vida diaria, desencadenando posicionamientos a favor (tecnófilos) o en contra (tecnófobos) de los nuevos cambios que condicionan y determinan la cotidianeidad ciudadana.

Entre los aspectos positivos cabe destacar la eminente posibilidad de tener acceso a la información desde cualquier punto del planeta, eliminando distancias y fronteras. No obstante, también se generan efectos negativos, como el aislamiento de las personas que están conectadas a la red durante todo el día y el consecuente detrimento en las relaciones interpersonales. Junto a los avances y posibilidades que nos ofrecen las nuevas tecnologías, también conllevan un riesgo que debemos afrontar de la manera más consecuente posible.

En este contexto, no podemos olvidarnos de los jóvenes, que mantienen una relación muy cercana con las TIC, gracias a las posibilidades que ofrecen, como la comunicación y el desarrollo de nuevas destrezas y habilidades. Así, Prensky (2011) denomina como "nativos digitales" a los niños nacidos y desarrollados con estas tecnologías, que las emplean con una naturalidad diferente al resto de generaciones, siendo capaces de manejar diferentes dispositivos sin que nadie les instruya.

Por otra parte, basándonos en las afirmaciones de Bringué y Sádaba (2009), observamos que nos encontramos ante una generación equipada, móvil, precoz, multitarea y emancipada. En todas las viviendas encontramos multitud de dispositivos de acceso a Internet, convirtiéndolas prácticamente en "ciberhogares". Los dispositivos móviles ya son imprescindibles, los jóvenes se desarrollan rápidamente y acceden sin problema a estas tecnologías, pueden prestar atención a la pantalla mientras realizan otras tareas y acceden a Internet desde sus dormitorios, generalmente en solitario y sin la supervisión de un adulto, lo que genera un riesgo para ellos.

Por otra parte, según Castells (2012) el cambio social más profundo en Internet se produjo en la primera década del siglo XXI con la interacción individual y corporativa. La llamada conexión inalámbrica conecta dispositivos, datos, gente u organizaciones con la nube, como si se tratara de un archivo de una red social generalizada, que todo lo cubre. Así, la actividad más importante en Internet actualmente pasa por los servicios de redes sociales (SNS), que se han convertido en plataformas para todo tipo de actividad, relaciones sociales, marketing, comercio electrónico, educación, creatividad cultural, distribución de los medios de comunicación, etc.

Los SNS son espacios vivos que conectan todas las dimensiones de la vida de la gente, lo que dota a la sociedad de una importante tendencia de transformación de la cultura, induciendo una cultura de compartir información. Por lo que, basándonos en las afirmaciones de Echeburúa y De Corral (2010), las motivaciones de los jóvenes para adquirir un móvil de última generación son múltiples y muy diversas: Ser visibles ante los demás, reafirmar la identidad ante el grupo o estar conectado con los amigos. El anonimato produce terror, al igual que asusta la soledad.

Los usos que los menores hacen de las TIC son amplios y diversos, ya que ofrecen múltiples posibilidades relacionadas con la búsqueda de información, distintos tipos de entretenimiento, comunicación, participación e incluso consumo. Por otra parte, la utilización de las redes sociales es fundamental y sirven como complemento a la comunicación presencial, donde la virtualidad adquiere mayor importancia. Este aspecto aporta una nueva dimensión a las redes sociales y las distingue del resto de herramientas y medios de comunicación.

Esta situación debe preocuparnos cuando el abuso de la tecnología provoca aislamiento, afecta a la autoestima y hace perder al sujeto su capacidad de control, generando incluso adicción. Lo que 
caracteriza a una adicción es la dependencia y la pérdida de control. La ciberadicción podría aparecer cuando el joven deja de verse con sus amigos y se instala frente a una pantalla, prestando más atención a su dispositivo móvil que al resto del mundo. En todos estos casos hay una interferencia claramente negativa en la vida cotidiana, que puede afectar a las relaciones personales del sujeto (Estallo, 2001).

Según Marcos (2013), los riesgos derivados de un mal uso de las TIC por parte de los jóvenes surgen en relación a dos ejes: las utilizan periódicamente y existe una evidente carestía respecto a medios de concienciación. Así, la falta de información y control puede exponer a los jóvenes a muchas de las amenazas existentes en la red, ya que de forma paralela a todos los avances que han supuesto las TIC, surgen también infinidad de amenazas, tales como el "phishing" (solicitud ilegal de claves), el "cyberbulling" (acoso a través de la Red), el "sexting" (envío mediante el teléfono móvil de imágenes con contenido sexual) o el "grooming" (suplantación de identidad por parte de adultos que se hacen pasar por menores).

Según Garmendia, Garitaonandia, Martínez y Casado (2011), el que más afecta a los menores entre 10 y 16 años es el contacto a través de Internet con personas que no conocen previamente. No se observan diferencias en cuanto al género en estas conductas. Resulta relevante destacar que la mayoría de los menores que acudieron a una cita con un desconocido contactaron con él a través de redes sociales. Por otro lado, el $10 \%$ de los menores aseguraron ser víctimas de problemáticas como el uso indebido de datos personales o el acceso a contenidos inapropiados generados por otros usuarios. Si bien señalan que el problema que más les afecta es el robo de contraseñas (8\%). Estos datos están en consonancia con las conclusiones establecidas por el estudio realizado por Díaz-Aguado, Martínez y Martín (2014) donde establecen que la mayoría de los jóvenes entrevistados aseguran que el riesgo en internet es "escaso".

Las denuncias por injurias y amenazas en las redes sociales son cada vez más frecuentes en los órganos judiciales españoles. Los procedimientos incoados por hechos ilícitos relacionados con las TIC se han incrementado en un 70\%, cifra que, según la última Memoria Anual de la Fiscalía General del Estado (2017), datos que refuerzan una realidad en constante evolución si lo comparamos con los datos del año 2016 , en torno al $64,40 \%$. Las denuncias suelen realizarse ante las autoridades, aunque redes sociales como Facebook o Twitter, también ponen a disposición de sus usuarios mecanismos para revelar estas situaciones, en caso de haber sido víctimas de injurias, calumnias o acoso. Asimismo, existen distintas páginas de denuncia como AlertCops (servicio de alertas de seguridad ciudadana de las Fuerzas y Cuerpos de Seguridad del Estado) o la Fundación ANAR (Ayuda a niños y adolescentes en riesgo), que ofrecen respuesta inmediata a problemas derivados del mal uso de las TIC. Sin embargo, los expertos advierten que todavía existe un número elevado de jóvenes que no denuncia este tipo de situaciones por miedo, vergüenza, o porque no conocen la existencia de estos canales. Estos datos ponen en evidencia una necesidad educativa, para que los jóvenes internautas conozcan sus derechos y los medios existentes para ser defendidos.

En el año 2009, el secretario de estado para las Telecomunicaciones, señalaba que el sistema educativo español debería contar con una asignatura para enseñar a los jóvenes a navegar por Internet con seguridad (Flores, 2009). Por otra parte, Gamito, Aristizabal, Vizcarra y Tresserra (2017), reflexionaron sobre la necesidad de empoderar a la población menor de edad para que puedan desenvolverse en el mundo online sin problemas, de manera eficiente, con el objetivo de superar las amenazas existentes. En esta misma línea se mostraba Aguayo (2016), para quien, dado que todos los niños deben pasar al menos 10 años de sus vidas en las escuelas, era una oportunidad para educar en principios, valores y habilidades para una convivencia positiva donde las TIC tengan un papel protagonista. Así, el reto desde las escuelas es múltiple. Por un lado, deben revisarse los fines, prácticas y metodologías educativas que puedan constituir formas de reproducción social de las causas de la violencia estructural y desarrollar un modelo de convivencia en positivo, y por otro, desarrollar planes de prevención de riesgos en el uso de las TIC, fomentando un uso responsable y crítico de las mismas. 
No debemos olvidar que el reto que se plantea a las escuelas y hasta ahora desarrollado de manera informal, no implica que no pueda llevarse a cabo en un contexto reglado, debido a los avances tecnológicos y a la era de la información y la comunicación en la que vivimos y de la que cada vez más niños y adolescentes forman parte.

\section{Diseño de investigación}

\subsection{Objetivos}

El aumento de casos de jóvenes que sufren problemas derivados de un mal uso de estas tecnologías, llegando a ser víctimas de suplantación de identidad o distintos tipos de acoso, nos llevó a preguntarnos si realmente son conscientes del uso que hacen de Internet y las redes sociales, planteando así este estudio que pretende averiguar su nivel de conocimiento y percepción mediante sus propias historias de vida.

Los objetivos de esta investigación se concretan en:

- Analizar el uso que hacen los jóvenes de Internet y redes sociales en un entorno hiperconectado.

- Conocer y comprender cuál es su percepción sobre los riesgos del uso de Internet y las redes sociales.

- Valorar la posibilidad de que se eduque de manera formal en un uso adecuado de estas tecnologías.

\subsection{Método}

Para el desarrollo de nuestra investigación, hemos utilizado el método biográfico-narrativo (Hallqvist y Hyden, 2013; Duncan, 2015) una modalidad de investigación que nos permitirá ampliar el conocimiento sobre la realidad de los jóvenes en relación al uso de internet y redes sociales, a través de sus propias experiencias y vivencias. Se pretendía así conseguir una posibilidad metodológica "más natural, accesible y democrática, estableciendo otro modelo de relación entre sujeto e investigador" (Rivas Flores, 2009, p.20). Para ello, hemos seguido la modalidad de entrevista narrativa seleccionado una serie de ítems, a modo de guion, que permitiesen, conducir y centrar inicialmente a los sujetos en sus experiencias concretas, sin limitar la información que nos quisieron transmitir (Anexo I).

\subsection{Procedimiento}

Se seleccionó un grupo de alumnos del ciclo de grado superior en Animación Sociocultural y Turística de un centro educativo de Salamanca subvencionado por la Junta de Castilla y León. Tras concertar una entrevista previa con uno de sus responsables, nos facilitaron los datos de contacto de diez alumnos, elegidos al azar, únicamente teniendo en cuenta el rango de edad entre 18 y 25 años. Una vez obtenidos los datos nos pusimos en contacto con ellos y tras una reunión previa en la que explicamos las características de la investigación, todos los alumnos accedieron a participar en el estudio, para ello firmaron un consentimiento informado que todos los participantes debieron leer y firmar antes de comenzar.

Las aportaciones de todos los informantes han sido tenidas en cuenta y consideradas en su totalidad. Todas las entrevistas han sido concertadas con anterioridad estableciendo fecha y hora para el desarrollo de las mismas. Todos los relatos biográficos se obtuvieron durante el mes de abril de 2018. Los encuentros tuvieron una duración aproximada de entre 30 y 40 minutos. Se realizaron de manera independiente y el espacio físico elegido para las mismas fue una sala destinada a reuniones, donde los participantes se sentían en mejor disposición para aportar su experiencia personal.

Todas las entrevistas fueron grabadas en su totalidad y posteriormente transcritas. Por último, cabe resaltar que de los análisis realizados se han establecido cuatro categorías principales: Acceso a internet y frecuencia de conexión, Conocimiento de las redes sociales y su utilización, Conocimientos sobre las 
influencia y los riesgos de la navegación en Internet y las redes sociales y Formación y necesidad de educar en las aulas en el uso adecuado de Internet y las redes sociales.

A continuación, se muestra un cuadro que refleja los datos e información más relevante de los alumnos que participaron en nuestra investigación. Para respetar cualquier dato identificativo todos los entrevistados son denominados con un pseudónimo.

\begin{tabular}{|c|c|c|c|c|c|}
\hline & SEXO & $\begin{array}{c}\text { AÑ̃o dE } \\
\text { NACIMIENTO }\end{array}$ & EDAD & $\begin{array}{l}\text { ACCESO A } \\
\text { INTERNET }\end{array}$ & $\begin{array}{c}\text { PERFIL EN } \\
\text { REDES } \\
\text { SOCIALES }\end{array}$ \\
\hline DAVID & Hombre & 1995 & 22 AÑOS & SI & NO \\
\hline VÍCTOR & Hombre & 1999 & 18 AÑOS & SI & SI \\
\hline BEATRIZ & Mujer & 1992 & 25 AÑOS & SI & SI \\
\hline ENRIQUE & Hombre & 1996 & 21 AÑOS & SI & SI \\
\hline LARA & Mujer & 1993 & 24 AÑOS & SI & SI \\
\hline SOFÍA & Mujer & 1996 & 21 AÑOS & SI & SI \\
\hline ANTONIO & Hombre & 1994 & 23 AÑOS & SI & SI \\
\hline SERGIO & Hombre & 1996 & 21 AÑOS & SI & SI \\
\hline MARÍA & Mujer & 1997 & 20 AÑOS & SI & SI \\
\hline SARA & Mujer & 1998 & 19 AÑOS & SI & SI \\
\hline
\end{tabular}

Figura 1. Alumnos/as participantes en el estudio.

\section{Resultados}

\subsection{Acceso a internet y frecuencia de conexión}

En cuanto a los dispositivos de acceso a Internet, ocho de los diez entrevistados afirman conectarse diariamente desde sus teléfonos móviles y ordenador. Antonio y Enrique apuntan también otros dispositivos.

- "Suelo conectarme desde el móvil, desde la Tablet cuando no estoy con el móvil. Con el ordenador, la verdad que bastante poco, en contadas ocasiones" (Enrique)

- "Normalmente me conecto con el móvil y sino, con la Tablet y el ordenador" (Antonio)

También encontramos casos, como el de Beatriz, en el que el uso del teléfono viene dado por la falta de otros dispositivos.

- "Me conecto únicamente desde el teléfono móvil, todos los días, no tengo ordenador" (Beatriz)

Respecto a la frecuencia de conexión, todos los entrevistados aseguran permanecer conectados durante la mayor parte del día. Además, coinciden en que la frecuencia de conexión desde sus ordenadores es mucho menor y con fines académicos. En este sentido, la excepción viene dada por Antonio quien solo utiliza los datos de su terminal móvil para consultar alguna información importante.

- "Normalmente me conecto desde el móvil todos los días, con el tema del Whatsapp. Con el ordenador me conecto con bastante menos frecuencia porque solo lo utilizo para hacer trabajos y cosas de clase" (Sergio)

- "Me conecto a la red con el móvil solo cuando estoy en casa, si utilizo los datos es porque se que me van a mandar un mensaje, pero no lo suelo tener activado por la calle" (Antonio) 
- "En Whatsapp y redes sociales estoy conectada todo el día, para hablar con la gente y comunicarme" (Sara)

- "Durante todo el día en el Whatsapp y las redes sociales para hablar con mis amigos o con mis familiares" (Beatriz)

Como podemos comprobar, la utilización de Whatsapp y de redes sociales resulta la principal finalidad de la conexión de los participantes. Otra finalidad de uso la aporta David, quien asegura que su alto grado de conexión es destinado a la búsqueda de información.

- "Permanezco conectado gran parte del día con la finalidad de buscar cosas de interés tanto a nivel formativo, como a nivel personal porque me gusta estar enterado de lo que ocurre en el mundo" (David).

\subsection{Conocimiento de las redes sociales y su utilización}

Todos los entrevistados afirman conocer redes sociales como Facebook, Instagram, Twitter, Snatchap y Tuenti. Alguno de los jóvenes consultados, como Sara o Antonio, mencionan otras redes sociales utilizadas principalmente para encontrar pareja. Lo más llamativo es que solo uno de los jóvenes consultados, David, asegura conocer una red de índole profesional.

- "Conozco, Facebook, Twitter, Instagram, Snatchap, Badoo, Tínder..." (Sara)

- "Conozco Facebook, Twitter, Instagram, Snatchap, Whatsapp, Badoo, Tínder, y seguro que alguna más pero ahora no me suena" (Antonio)

- "Conozco Twitter, Facebook, Instagram, LinkedIn, Tuenti (aunque ya no se utiliza) y no sé, ahora no me acuerdo de ninguna más" (David)

Respecto al uso de las redes sociales, únicamente David refiere no manejar ninguna de ellas, en el resto de los casos consultados, todos tienen más de un perfil activo en distintas redes sociales. Las más habituales son Instagram y Facebook en 9 de los 10 entrevistados. Solo Enrique tiene además una cuenta en Twitter, una red cada vez más en desuso entre los usuarios jóvenes.

- "Ahora mismo solo utilizo Whatsapp si se puede considerar red social, porque el resto de redes no las tengo. Lo utilizo para comunicarme y para estar enterado a nivel de clase porque tenemos un grupo, y también lo utilizo para quedar con mis amigos" (David)

- "Sí, yo tengo Instagram y Twitter para enterarme de las noticias, pero casi no lo utilizo" (Enrique)

En cuanto a la finalidad de uso dado a las redes sociales, encontramos que el motivo principal es poder comunicarse, especialmente con amigos, subir, ver y enviar material gráfico y compartir información. Sofía y Antonio reconocen otros usos.

- "Sí utilizo redes sociales, sobre todo Facebook e Instagram, y la verdad es que principalmente las utilizo para cotillear" (Sofía)

- "Sí las utilizo, sobre todo el Facebook para informarme de noticias deportivas, o también lo utilizo para cotillear a algún amigo. Y el Instagram, la verdad que yo no suelo subir nada, pero reconozco que lo utilizo para cotillear y estar enterado de las cosas" (Antonio)

En relación a la edad a la que comenzaron los participantes a utilizar Internet y redes sociales, oscila entre los 13 y los 16 años, coincidiendo con el comienzo de la Educación Secundaria. El caso de David es interesante, ya que además aporta información sobre su decisión de dejar de utilizarlas.

- "Yo creo que empecé sobre los 13 años, antes sí que usaba redes sociales y sobre esa edad utilizaba el mesengger. Ahora ya no las tengo, la verdad es que ha cambiado mucho mi forma de ver las redes sociales respecto a esta época y por eso, entre otras razones he decidido quitarlas" (David) 


\subsection{Conocimientos sobre la influencia y los riesgos de la navegación en Internet y las redes sociales.}

Respecto a la influencia que genera internet y las redes sociales, todos los participantes del estudio refieren una influencia negativa salvo Enrique, que destaca algún aspecto positivo. Antonio es más concreto y añade el impacto que tiene sobre la autoestima, mientras que Beatriz recalca también la influencia en el rendimiento escolar.

- "Yo creo que ahora la gente está super enganchada a las redes sociales, y que lo utilizamos más que para informarnos de las noticias, para cotillear, para ver qué pasa o de que chisme me entero. Creo que a la larga, la sociedad va estar desinformada de lo que ocurre realmente, porque estaremos metidos en una burbuja y no vamos a salir más allá del círculo de nuestros ciberamigos, porque se están perdiendo todas las relaciones interpersonales. Ahora para que una persona se sienta bien tiene que subir una foto en vez de comunicarlo cara a cara o llamar por teléfono, la gente necesita subir una foto y que esa foto reciba me gustas para sentirse bien" (Antonio)

- "Creo también que nos influye mucho en el rendimiento escolar, porque al estar conectados, muchas veces no prestamos atención en clase o a la hora de estudiar, por lo que considero que nos influyen de manera negativa en la mayoría de los casos" (Beatriz)

- "Depende, porque por ejemplo redes sociales como el Twitter puede ser positivo porque te enteras de todo lo que pasa en el mundo en tiempo real, pero también te pueden influir negativamente porque te pueden crear una adicción o te puede influir en tu forma de pensar. Y supongo que eso se puede extrapolar al resto de redes sociales, que todas tienen sus ventajas, pero también muchos inconvenientes" (Enrique)

En relación a la percepción del riesgo que tienen nuestros participantes cuando acceden a Internet y utilizan las redes sociales, afirman ser conscientes de los mismos, sin embargo los perciben como algo lejano.

- "Sí, soy consciente de que existen riesgos y peligros y que a muchas personas les pasan cosas, pero yo creo que como a mí no me ha pasado, pienso que nunca me van a suceder cosas malas y las sigo utilizando incorrectamente" (Beatriz)

- "Sé que existen riesgos y peligros, pero tampoco lo identifico como tal, porque si no, no utilizaría Internet ni redes sociales. Es decir, soy consciente, pero no le doy importancia" (Sara)

Resulta interesante resaltar el caso de Antonio, ya que es el único que intenta impedir este tipo de peligros.

- "Sí, riesgo hay cada vez que te conectas a Internet, aunque yo la verdad que todas las redes sociales las tengo lo más privadas posible, si alguien quiere seguirme lo tengo que aceptar yo, primero veo quién es, si le conozco y si me interesa que esa persona me siga. Pero yo creo que hay muchas personas que lo único que les importa es que aumente el número de seguidores y no ven más allá de quién puede estar accediendo a su información" (Antonio)

Como podemos apreciar en este caso, Antonio procura mantener sus perfiles en Internet cerrados, sin embargo, es consciente de que muchos jóvenes no hacen lo mismo y afirma que para ellos es más importante tener una cuenta con percepción positiva a los riesgos que puedan correr al exponerse.

Precisamente, respecto a los riesgos y peligros, podemos observar como el ciberacoso, en sus distintas versiones, es mencionado por todos los participantes en sus discursos.

- "Lo que más el acoso, porque no sabes quién puede estar detrás de la pantalla y a lo mejor te piensas que es un amigo y no lo es. También ser que te pueden observar a través de la cámara web, porque te la pueden abrir sin darte cuenta" (Lara) 
- "Ciberbullying, distintos tipos de acoso, no solo a niños pequeños, sino gente que se hace pasar por otras personas y te intenta influenciar" (María)

Además del ciberacoso, también encontramos referencias a la pornografía infantil y el sexting. Por su parte, Sara comenta la suplantación de identidad o el robo de fotos personales como otros de los posibles riesgos. Antonio añade a los ya mencionados, la extorsión y el grooming.

- "Que te roben información que tú piensas que no te pueden quitar porque una vez que subes algo a Internet es de todos, también el tema de hackers y gente que te puede extorsionar por tener información tuya, y pedirte dinero. También el sexting, ciberbullying y había otro que no recuerdo muy bien el nombre, grooming ¿puede ser?" (Sara)

Por otra parte, ninguno de los entrevistados ha vivido en primera persona una experiencia relacionada con los peligros mencionados. Sin embargo, seis de ellos sí conocen a alguna persona que ha sido víctima.

- "Víctima no he sido, conocer sí que conozco a gente que ha podido sufrir un acoso mediante Internet, porque se han metido con ellos, les vacilan por los grupos de Whatsapp, y al principio parece que es una tontería, pero se han terminado cansando y lo pasan mal" (Enrique)

- "Yo no he sido víctima, pero conozco el caso de una chica que le insultaban y se metían con ella por el Facebook y se reían de ella, y esta chica lo pasó bastante mal y se tuvo que cambiar de instituto" (Sergio)

- "Víctima no he sido, pero algún compañero mío que por bobadas ha podido insultar a otro por las redes sociales y esto ha traído después problemas más graves que le han afectado en su vida personal" (Víctor)

En relación a las consecuencias en el caso de cometer una infracción relacionada con las redes sociales e internet, cuatro de los participantes afirman desconocer totalmente los efectos que acarrea. El resto muestra cierto conocimiento, aunque no profundizan en ello.

- $\quad$ "Imagino que en primera instancia pueden ponerte una multa, y dependiendo de la gravedad del asunto hasta pena de cárcel, por ejemplo, en el caso de pederastas o acosadores de menores" (Sergio)

- "Supongo que multa, bloqueo de cuenta o denuncia" (Sara)

- "Sí, que te multan o que te pueden meter en la cárcel" (Enrique)

- "Más o menos, sé que está penado, pero supongo que depende si la infracción es cometida por un menor de edad o no. En el caso de los menores de edad, escuché una conferencia de Emilio Calatayud que les imponía castigos prácticos por así decirlo, y en el caso de los mayores supongo que también depende de la infracción, pero no lo tengo muy claro" (David)

La mayor parte de los jóvenes consultados entienden que cometer una infracción de esta índole conlleva implicaciones legales. Mencionan multas, bloqueos de cuentas e incluso la privación de libertad. En este sentido, son conscientes de que este tipo de actuaciones tienen consecuencias. Por otra parte, es llamativo que cuatro de los diez entrevistados desconozcan totalmente cuáles son estas implicaciones.

\subsection{Formación y necesidad de educar en las aulas en el uso adecuado de Internet y las redes sociales}

En relación a la formación recibida en esta materia, siete de los participantes mencionan charlas puntuales en el instituto, en las que pretendían concienciarles de esta problemática a través de pequeños discursos. El resto alude no recordar ningún tipo de formación. 
- "Una vez una charla de 30 minutos, pero tampoco te explicaban mucho, que son malas, que no subas fotos, que si las subes ten cuidado con lo que subes, lo típico" (Sara)

- "Formación como tal no, igual comentarnos por encima entre clase y clase que uso inadecuando hay de Internet, pero como tal una formación no" (Antonio)

- "En el instituto nunca he recibido una formación de este tipo, solo algún curso que he realizado yo por mi cuenta" (Beatriz)

Respecto a la percepción de los participantes sobre la importancia de incluir formación específica en los centros educativos, todos ellos coinciden en que es fundamental que desde la escuela se trabaje la educación para el buen uso de las nuevas tecnologías.

- "Sí, porque actualmente los jóvenes no saben los riesgos que conlleva el uso de Internet y a que están expuestos, y con la educación en las aulas podrían evitarse muchas situaciones que se dan por falta de información" (Lara)

- "Sí, yo creo que es muy importante, porque si desde pequeño te prohíben las cosas sin explicártelas, la tentación te puede y vas a entrar en Internet. Sin embargo, si nos formaran para utilizarlo y nos enseñara a hacerlo de forma adecuada y responsable creo que no habría ningún problema." (María)

Sin embargo, algunos de los entrevistados, como Antonio, Enrique o Beatriz, van un paso más allá y aseguran que se debe incluir este tipo de formación desde el inicio de la escolarización.

- "Sí, yo creo que sí, pero más allá de recordarlo en los institutos, yo creo que habría que hacerlo en los colegios, cuando empiezas a utilizar el móvil y las redes sociales, así enseñarías a hacer un uso correcto desde el principio, porque una vez que se empieza a utilizar mal, es muy difícil cambiar" (Enrique)

- "Creo que sí es necesaria la formación y, sobre todo, considero importante empezar cuando somos más pequeños y empezamos a utilizar Internet, porque es la base y si empiezas utilizándolas mal es muchísimo más difícil cambiar los hábitos" (Antonio)

- "Sí, considero que en todos los institutos e incluso en los colegios desde bien pequeños deberían explicar un uso adecuando tanto de Internet como de las redes sociales, porque si no, muchos niños no se dan cuenta del tipo de fotos que suben o incluso de no tener privadas las cuentas y hacen cosas sin pensar que a la larga pueden tener consecuencias" (Beatriz)

Haciendo referencia a la capacidad preventiva de la educación a la hora de evitar problemas en la utilización de internet y las redes sociales, todos los participantes la consideran un instrumento de concienciación necesario para una utilización adecuada.

- "Yo creo que sí porque los niños son como esponjas, entonces si tú les enseñas que hay unas acciones que si las hacen les van a perjudicar, yo creo que se van a quedar con ello y van a actuar de diferente manera" (Antonio)

- "Sí, porque pienso que, si los profesores nos enseñaran a utilizar bien las redes sociales, no solo para no hacer cosas que perjudiquen a otras personas, sino para aprender que las redes sociales sirven para comunicarnos, aprender de las experiencias de vida de otras personas etc., el uso que le daríamos sería mucho mejor" (Víctor)

Sin embargo, Sara se muestra más reticente al cambio, aunque está de acuerdo con la premisa de partida.

- "Creo que es muy difícil evitar estas situaciones porque hoy en día todo el mundo utiliza Internet y las redes sociales, y por mucho que nos digan no vamos a dejar de hacerlo. Creo que 
aprenderemos el día que nos pase alguna de estas situaciones, pero sí que pienso que, si te enseñan a utilizarlas bien, se podrían disminuir los casos derivados de un mal uso de Internet" (Sara)

Todos los jóvenes consultados coinciden en que, gracias a una formación adecuada en este ámbito, se podrían evitar o al menos disminuir el ciberbullying. Solo Víctor incide en otras realidades.

- "Creo que el ciberbullying sobre todo, porque es el mayor tipo de acoso y a través de la educación estaríamos mucho más mentalizados sobre lo que puede pasar" (Sofía)

- "El ciberbullying, las amenazas un poco lo que hablábamos antes. Pero sobre todo creo que podría evitarse el acoso escolar, porque cuando empieza no se acaba en clase, el chaval sigue recibiendo acoso por parte de sus compañeros y este aumenta en las redes sociales, mediante fotos, insultos etc., por lo que creo que en este tipo de casos la educación es totalmente necesaria" (David)

- "El ciberbullying, el mal uso de las TIC, y distintos tipos de discriminación, como la violencia de género, racismo (porque muchas veces se ve por las redes sociales insultos a personas que no son como nosotros, etc.)" (Víctor)

\section{Discusión de los resultados}

La incorporación de las nuevas tecnologías a nuestras vidas nos muestra el rápido avance de las mismas como herramienta de interacción social, pero también como medio de aprendizaje, satisfacción de la curiosidad o diversión (Castellana, Sánchez Carbonell, Graner y Beranuy, 2007). Esta realidad nos lleva a ser conscientes de que Internet y las redes sociales, además de ofrecer diferentes oportunidades a las nuevas generaciones, también conllevan numerosos riesgos derivados de su utilización.

Como hemos podido comprobar a lo largo del estudio, los jóvenes participantes en la presente investigación manifiestan hacer un uso elevado de Internet y de las redes sociales, estando conectados prácticamente todo el día. Estos datos están en consonancia con investigaciones que muestran tasas por encima del $90 \%$, fundamentalmente en adolescentes, con objetivos de carácter comunicativo (Fernández-Montalvo, Peñalva e Irazabal, 2015).

Por otra parte, en relación a los dispositivos de acceso a Internet y a las redes, los participantes resaltan la utilización, en mayor medida, de dispositivos que permiten la conexión a la red desde cualquier lugar y en cualquier momento (smartphones). Estos dispositivos, han cobrado protagonismo respecto a otros como pueden ser los ordenadores o las tablets. Como consecuencia, los jóvenes viven en un periodo de conectividad permanente, el cual provoca que estén continuamente activos en Internet. Estudios como el desarrollado por Ruíz-Palmero, Sánchez-Rodríguez y Trujillo-Torres (2015), establecen que el 90,7\% de adolescentes acceden a internet mediante el teléfono móvil, siendo además una seña de identidad que favorece una independencia comunicativa.

Por otro lado, todos los participantes en el estudio comenzaron a utilizar Internet y las redes sociales a edades muy tempranas, situando la media de acceso entre los 13 y los 16 años. Estos datos coinciden con los aportados por Urdín, Perurena, Arcos y Morentin (2013), que refieren que existe un $73 \%$ de adolescentes entre 13 y 15 años que usan las redes sociales. Si a estas circunstancias unimos la falta de información y experiencia vital, estamos ante un colectivo vulnerable en la red.

En cuanto a las redes sociales utilizadas, los jóvenes consultados mencionan principalmente herramientas para mantener contacto con otras personas, subir y visualizar fotografías. Las más comunes son Facebook, Instagram, Snatchap y Whatsapp y rara vez Twitter u otras aplicaciones que permiten el 
intercambio de información. No debemos olvidar que Según Bernal y Angulo (2012), el uso de las redes sociales suponen un medio de socialización por parte de los jóvenes, por lo que tiene sentido que, herramientas y redes destinadas a la comunicación y al intercambio y visualización de fotografías, sean medio fundamental para ampliar o mantener la red de contactos. Asimismo, es importante destacar que tan solo uno de los jóvenes entrevistados no dispone de cuenta en ninguna red social, hecho que resulta llamativo, teniendo en cuenta la magnitud que están cobrando este tipo de herramientas en el día a día de los jóvenes.

A pesar de los datos expuestos, todos estos jóvenes manifiestan que Internet y las redes sociales están influyéndoles de forma negativa, tanto a la hora de comunicarse con los demás como en otros aspectos, como el rendimiento académico. También refieren efectos negativos en la propia autoestima, una consecuencia que ha sido corroborada por autores como Niemz, Griffiths y Banyard (2005).

Por otra parte, a la hora de identificar riesgos y peligros derivados de un mal uso de Internet y las redes sociales, se pone de manifiesto, por parte de los participantes, que a pesar de ser conscientes de que ocurren situaciones negativas por no utilizar de forma correcta Internet y redes sociales, siguen haciendo un uso indebido. En este sentido, los riesgos más conocidos son los distintos tipos de acoso, aunque aseguran también conocer otros como la pornografía infantil, sexting, la suplantación de identidad o el robo de fotos personales, la extorsión o el grooming, riesgos que coinciden con los destacados por Gamito, Aristizabal y Olasolo (2017). Estos datos no resultan extraños si tenemos en cuenta la encuesta sobre hábitos de uso y seguridad de internet de menores y jóvenes en España (2014), donde se establece que existe un uso masivo de Internet y redes sociales por parte de los jóvenes. Además, un tercio de los contactos que tienen los menores en Internet son de personas que no conocen personalmente y donde existe una subida generalizada de fotos y videos a la red (Ministerio del Interior, 2014), sin ser conscientes de las situaciones y peligros del uso sin su conocimiento (García-Jiménez, 2011).

Siguiendo con los testimonios de los participantes, en ninguno de los casos han sido víctimas de los riesgos mencionados en líneas anteriores, pero llama la atención que a pesar de que la mayoría de los jóvenes conocen víctimas de algún tipo de experiencia negativa en Internet y en redes sociales, no tienen una idea definida acerca de si el uso de dichas herramientas conlleva riesgos hacia su persona y lo perciben como algo lejano. Esta realidad está en consonancia con las aportaciones de García-Jiménez (2011), que estableció en un estudio sobre los riesgos que sufren los adolescentes en Internet, que un $19,5 \%$ no se ha llegado a cuestionar los peligros que puede representar el acceso al ciberespacio, un $39,5 \%$ navega totalmente seguro y un $33,8 \%$, relativamente seguro, lo que denota una ausencia de percepción sobre los peligros existentes en la red.

En cuanto a las implicaciones legales en el uso apropiado de Internet y redes sociales, la mayoría de los jóvenes que formaron parte de nuestro estudio entienden que cometer una infracción conlleva unas implicaciones legales $y$, aunque no tienen muy claras cuáles son, sí que son conscientes de que este tipo de actuaciones, a través de Internet y redes sociales, tienen consecuencias. Sin embargo, desconocen totalmente cuáles son los canales de denuncia en caso de vivir una situación similar y eso también puede ser debido a la baja percepción del riesgo que hemos comentado anteriormente y que genera que "muchos jóvenes no vean las consecuencias de volcar en las redes sociales toda su privacidad e intimidad" (Mata, 2017, p.19).

Por último, en cuanto a la formación recibida y la necesidad de educar en las aulas en el uso adecuado de Internet y las redes sociales y los riesgos que pueden surgir, los jóvenes consultados manifiestan no haber recibido ninguna formación reglada a lo largo de su etapa educativa. La única formación que han recibido en esta materia ha sido mediante pequeñas charlas puntuales en los centros educativos, impartidas muchas veces por profesionales ajenos al ámbito educativo, como por ejemplo policías. A su vez, todos los jóvenes entrevistados consideran totalmente necesaria la educación en las aulas a lo largo 
de toda la etapa educativa. Además, aseguran que es fundamental que esta educación se inicie en el momento en que los alumnos/as comienzan a utilizar las TIC, ya que, mediante la prevención, podrían introducirse buenos hábitos de uso, que son más difíciles de modificar cuando ya son usuarios habituales.

Precisamente, autores como Gamito, Aristizabal y Olasolo (2017) refieren que contenidos relacionados con la gestión de la privacidad o la vivencia en Internet deben formar parte de los planes educativos ya que las tecnologías por sí solas, no ayudan ni aconsejan al alumnado (Viñals y Cuenca, 2016). Por todo ello, no debemos olvidar que el principal objetivo del sistema educativo actual consiste en formar ciudadanos que puedan adecuarse a una sociedad en constante cambio, caracterizada por la información y el conocimiento. Sin olvidarnos de que la competencia digital es una competencia clave para el desarrollo personal y social de los individuos.

\section{Conclusiones}

Para concluir debemos resaltar que el presente artículo, ha contribuido a evidenciar el cumplimiento de los tres objetivos propuestos. Además, dichos objetivos, están en consonancia con la contribución realizada a la comunidad científica. Se han analizado los hábitos en el uso de internet y las redes sociales en un grupo de jóvenes a través de sus relatos vitales, un uso excesivo y realizado fundamentalmente a través del smartphone. Además, también se ha reflejado la percepción sobre los riesgos en el uso de internet y las redes sociales, un riesgo caracterizado por ser escaso a pesar de reconocer la existencia de consecuencias derivadas de su mala utilización. A pesar de ello, existe unanimidad respecto a la necesidad de incluir en el ámbito educativo una formación específica, desde edades tempranas, orientada a mejorar el conocimiento y el uso adecuado tanto de internet como de las redes sociales.

Respecto a las limitaciones, destacar que la muestra podría haber sido más amplia. También se podrían haber seleccionado otros rangos de edad para comprobar las diferencias en el uso de internet y las redes sociales en función de dicho criterio. Por otra parte, una muestra más grande, habría permitido utilizar otro tipo de metodología o incluso un método mixto.

Por último, las líneas futuras deben ir orientadas a realizar estudios longitudinales que permitan poder evaluar, a lo largo del tiempo, el conocimiento y uso de internet y redes sociales que hacen los jóvenes. También se deben diseñar programas específicos que puedan incluirse en las escuelas con el propósito de conseguir una formación adecuada que permita una navegación segura a través de la red.

\section{Referencias bibliográficas}

Aguayo, A. (2016). Intervención Social con menores y TICs. Un modelo integral para la prevención de riesgos. Pedernal. Recuperado de http://pedernal.org/ebookintervencion-social-menores-tic/

Bernal, C., y Angulo, F. (2012). Interacciones de los jóvenes andaluces en las redes sociales. Comunicar, 20(40), 25-30.

Berríos, LI., \& Buxarrais, M. R. (2005). Las tecnologías de la información y la comunicación (TIC) y los adolescentes. Algunos datos. Monografías virtuales. Ciudadanía, democracia y valores en sociedades plurales. OEI, 5(5). 
Bringué, X,. \& Sádaba, C.H. (2009) La Generación Interactiva en España. Niños y adolescentes ante las pantallas. Barcelona: Ariel.

Castellana, M., Sánchez-Carbonell, X, Graner, C., \& Beranuy, M. (2007). El adolescente ante las tecnologías de la información y la comunicación: Internet, móvil y videojuegos. Papeles del Psicólogo, 28(3), 196-204.

Castells, M (2012). Redes de indignación y esperanza. Madrid. Alianza

Díaz-Aguado, M. J., Martínez, R., \& Martín, J. (2014). Evolución de la adolescencia española sobre la igualdad y la prevención de la violencia de género. Madrid: Ministerio de Sanidad, Servicios Sociales e Igualdad.

Duncan, S. (2015). Biographical Learning and Non-Formal Education: Questing, Threads and Choosing How to Be Older. Studies in the Education of Adults, 47(1), 35-48.

Echeburúa, E., \& De corral, P. (2010). Adicción a las nuevas tecnologías y a las redes sociales en jóvenes: un nuevo reto. Adicciones, 22(2), 91-95.

Estallo, J.A. (2001). Usos y abusos de Internet. Anuario de Psicología, 32, 95-108.

Fernández-Montalvo, J., Peñalva, A., \& Irazabal, I. (2015). Hábitos de uso y conductas de riesgo en Internet en la preadolescencia. Comunicar, 44(22), 113-120.

Flores, F. (2009). Uso seguro de Internet y ciudadanía digital responsable. Recuperado de: http://www.pantallasamigas.net/proteccion-infancia-consejos-articulos/pdfs/pantallasamigas-usoseguro-de-Internet-y-ciudadania-digital-responsable.pdf

Gamito, R., Aristizabal, P., \& Olasolo, M. (2017). La necesidad de trabajar los riesgos de internet en el aula. Profesorado. Revista de Currículum y Formación del Profesorado, 21(3), 409-426.

Gamito, R., Aristizabal, P., Vizcarra, M.T., \& Tresserras, A. (2017). La relevancia de trabajar el uso crítico y seguro de internet en el ámbito escolar. Fonseca, Journal of Communication, 14, 11-25.

García-Jímenez, A. (2011). Una perspectiva sobre los riesgos y usos de Internet en la adolescencia. Revista Icono, 14, 396-411.

Garmendia, M., Garitaonandia, C., Martínez, G., \& Casado, M. A. (2011). Riesgos y Seguridad en Internet: Los menores españoles en el contexto europeo. Resultados de la encuesta de EU Kids Online a menores de entre 9 y 16 años $y \quad a$ sus padres $y$ madres. Recuperado de: http://www.Ise.ac.uk/media@Ise/research/EUKidsOnline/EU\%20Kids\%20II \%20(200911)/National\%20reports/Spanish\%20report.pdf

Hallqvist, A., \& Hyden, L.-Ch. (2013). Work Transitions as Told: A Narrative Ap- proach to Biographical Learning. Studies in Continuing Education, 35(1), 1-16.

Marcos, M. (2013). La influencia de las TIC en el desarrollo del adolescente y preadolescente. Hacia una ciudadanía digital responsable. Valladolid. Universidad de Valladolid.

Martínez Pastor, E., García Jímenez, A., \& Sendín Gutierrez, J.C. (2013). Percepción de los riesgos en la red por los adolescentes en España: usos problemáticos y formas de control. Anàlisi Monogràfic, 48, 111130. 
Mata Mayrand, L. (2017). Aspectos jurídicos del acoso y ciberacoso escolar. Revista de Estudios de Juventud, 114, 13-19.

Memoria Anual de la Fiscalía General del Estado. (2017). Capítulo III. Criminalidad Informática. Recuperado de https://www.fiscal.es/memorias/memoria2018/FISCALIA_SITE/index.html

Ministerio de Interior (2014). Encuesta sobre hábitos de uso y seguridad de Internet de menores y jóvenes en España. Madrid. Recuperado de https://bit.ly/1x31JXy

Niemz, K., Grieffiths, M., \& Bayard, P. (2005). Prevalence of pathological Internet use among university students and correlations with self-esteem, the general health questionnaire (GHD), and disinhibition. Cyberpsychology \& Behavior, 8(6), 562-570.

Prensky, M. (2011). Enseñar a nativos digitales. España: Editorial SM.

Rivas Flores, J.I. (2009). Narración, conocimiento y realidad. Un cambio de argumento en la investigación educativa. En J.I. Rivas Flores y D. Herrera Pastor (coords.), Voz y Educación. La narrativa como enfoque de interpretación (pp. 17- 36). Barcelona: Octaedro.

Ruiz-Palmero, J., Sánchez-Rodríguez, J., \& Trujillo-Torres, J. M. (2016). Utilización de Internet y dependencia a teléfonos móviles en adolescentes. Revista Latinoamericana de Ciencias Sociales, Niñez y Juventud, 14(2), 1357-1369.

Urdín, J., Perurena, N., Arcos, A., \& Morentin, J. I. (2013). Las redes sociales y las diferencias intergeneracionales: un análisis entre Europa y Estados Unidos. Revista Fuentes, 13, 309-326. Recuperado el 10 de mayo de 2017 de: https://ojs.publius.us.es/ojs/index.php/fuentes/article/view/2581

Viñals Blanco, A. y Cuenca Amigo, J. (2016). El rol del docente en la era digital. Revista Interuniversitaria de Formación del Profesorado, 30(2), 103-114. 


\section{Anexo I}

\section{Guion de las entrevistas narrativas con los jóvenes participantes en el estudio}

1. ¿Desde qué dispositivos te conectas a la red y con qué frecuencia?

2. ¿Cuánto tiempo permaneces conectado a internet aproximadamente? ¿Con qué finalidad?

3. ¿En qué lugares accedes a la red con más frecuencia?

4. ¿Qué redes sociales conoces?

5. ¿Utilizas las redes sociales en tu día a día? ¿Con qué finalidad?

6. ¿A qué edad comenzaste a utilizar internet y las redes sociales?

7. ¿Cómo crees que influye el uso de internet y las redes sociales en los jóvenes y adolescentes?

8. ¿Identificas riesgo y peligros al usar internet y redes sociales?

9. ¿Qué riesgos presentes en la red conoces?

10. ¿Has sido víctima o conoces a alguna víctima de alguno de los riesgos mencionados?

11. ¿Conoces las implicaciones legales en el uso inapropiado de internet y las redes sociales?

12. ¿Conoces algún canal de denuncia para las víctimas de delitos en la red?

13. ¿Has recibido alguna formación reglada sobre el uso adecuado de internet y las redes sociales?

14. ¿Has recibido formación reglada sobre los riesgos que surgen el uso incorrecto de internet y las redes sociales?

15. ¿Consideras necesaria la educación en las aulas sobre el uso adecuado de internet y las redes sociales? ¿Por qué?

16. ¿Piensas que podrían evitarse situaciones desagradables, derivadas de un mal uso de internet y las redes sociales, si se educara en las aulas? ¿Por qué?

17. ¿Qué situaciones crees que podrían evitarse si desde los centros educativos se educara en el uso adecuado de internet y las redes sociales? 